\title{
Dynamic modeling and identification of the Uniovi structure
}

\author{
José Luis Zapico-Valle* and Marta García-Diéguez \\ Department of Construction and Engineering Manufacturing, University of Oviedo, Campus de Gijón 7.1.16, 33203 Gijón, Spain
}

Received 21 February 2013 / Accepted 13 November 2013 / Published online 6 February 2014

\begin{abstract}
The nonlinear modeling and identification of a four-storey steel frame is presented in this paper. The bending modes of the frame were experimentally isolated by a single-point mono-harmonic excitation and used for identification purposes. A novel expeditious procedure was developed to infer the kind of nonlinearities present in the structure. This turned out to be the most critical task in the modeling process. The proposed nonlinear model was calibrated in the time domain by fitting the model-predicted responses to the experiments. This was posed as the minimization of an error function by means of a new adaptive stochastic algorithm. Results were excellent. The calibrated nonlinear model yield fitting errors near three orders of magnitude lower than those of a pure linear model.
\end{abstract}

Key words: Nonlinear model, Nonlinear modal identification, Softening stiffness.

\section{Introduction}

Frame steel structures are extensively used in industrial and building construction. Linear models are usually adopted in structural applications as design or health monitoring. This approach strongly simplifies the modeling and extraction of modal properties. Linearity, however, seldom occurs in reality. Structures always contain some degree of nonlinearity due to material and interface behavior, geometric and elastic instabilities, etc. An epistemic error is therefore introduced by linear modeling and a nonlinear approach should be chosen when accurate predictions are required. Theory of nonlinear systems has evolved from the seminal work of Rosenberg [1] and is a mature subject nowadays. Kerschen et al. [2] and Peeters et al. [3] attempted to reconcile practice to nonlinear theory. The application of this theory, however, has been restricted to numerical simulations or small experimental rigs, in which the type of nonlinearity is known beforehand. The nonlinear theory has not yet been applied to full-scale engineering structures with multiple components. This paper deals with the nonlinear modal identification of a four-storey medium-size steel frame. Special emphasis is placed on the design of experiments and the subsequent inference of adequate nonlinear models. The aim of the study is to extract very accurate values of the parameters of the models, which will be the basis for future applications on finite element model updating and structural health monitoring.

\footnotetext{
*e-mail: jzapico@uniovi.es
}

\section{Experimental part}

\subsection{Structure}

The Uniovi structure is a middle-size four-storey steel frame with two bays in the longitudinal direction and one bay in transversal one. The overall dimensions of the structure are $4 \mathrm{~m}$ length, $1.5 \mathrm{~m}$ width and $7.3 \mathrm{~m}$ height (see Figure 1). All columns and beams are HEA-120 and IPN-100, respectively, of steel grade S-275. The floors of the frame are steel sheets $4 \mathrm{~mm}$ thick connected to the beams through discontinuous weld. The foundations are two continuous concrete beams lying on the floor of the laboratory.

Each column consists of two pieces. They are spliced through end plates connected by four bolts $12 \mathrm{~mm}$ in diameter. The columns are welded to $20 \mathrm{~mm}$ thick plates. The beams corresponding to the transversal direction are directly connected to the web of the columns by a welded-all-around fillet. In the longitudinal direction, however, the beams are connected to the flange of the columns. The connection was designed with a $8 \mathrm{~mm}$ thick end plate and four bolts $10 \mathrm{~mm}$ in diameter.

\subsection{Exciter and measurement chain}

A pendulum-like exciter was used to excite the frame. It consists of an eccentric mass connected to the shaft of a variable-speed electrical motor (see Figure 2). The excitation was carried out by harmonically varying the angular position of the mass. The frequency and amplitude of the excitation were achieved by controlling the angular position of the shaft 


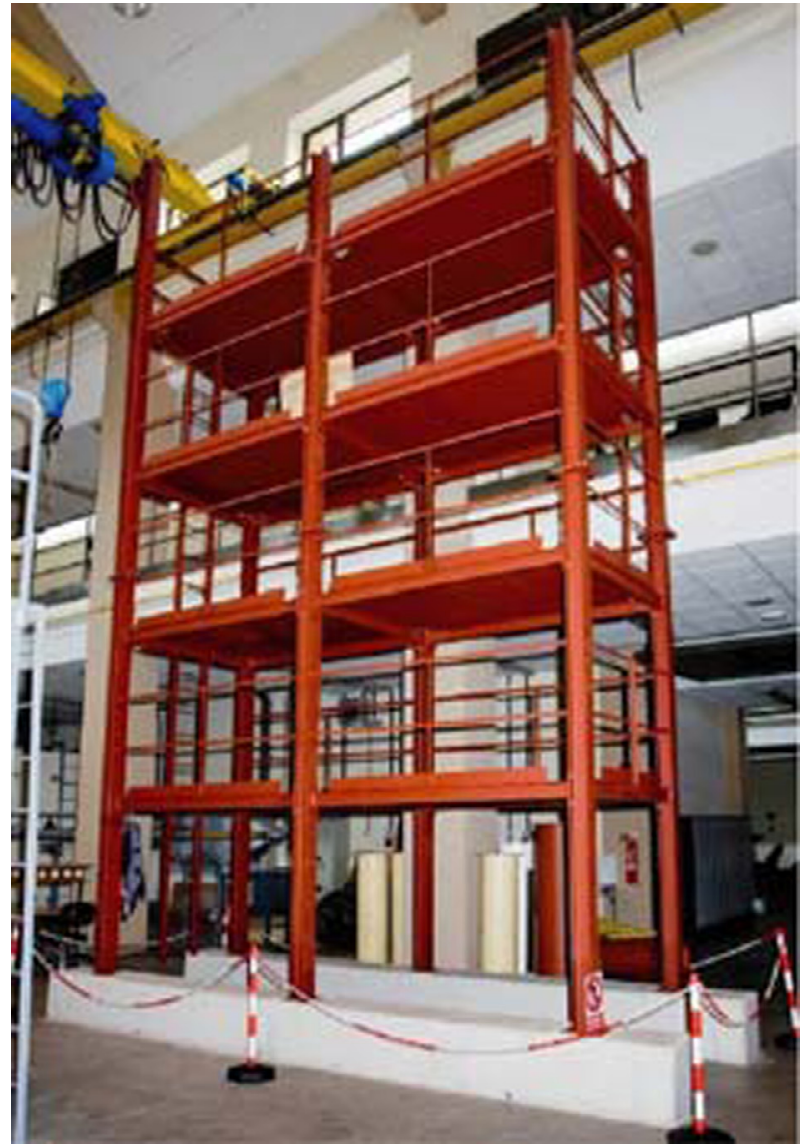

Figure 1. Structure.

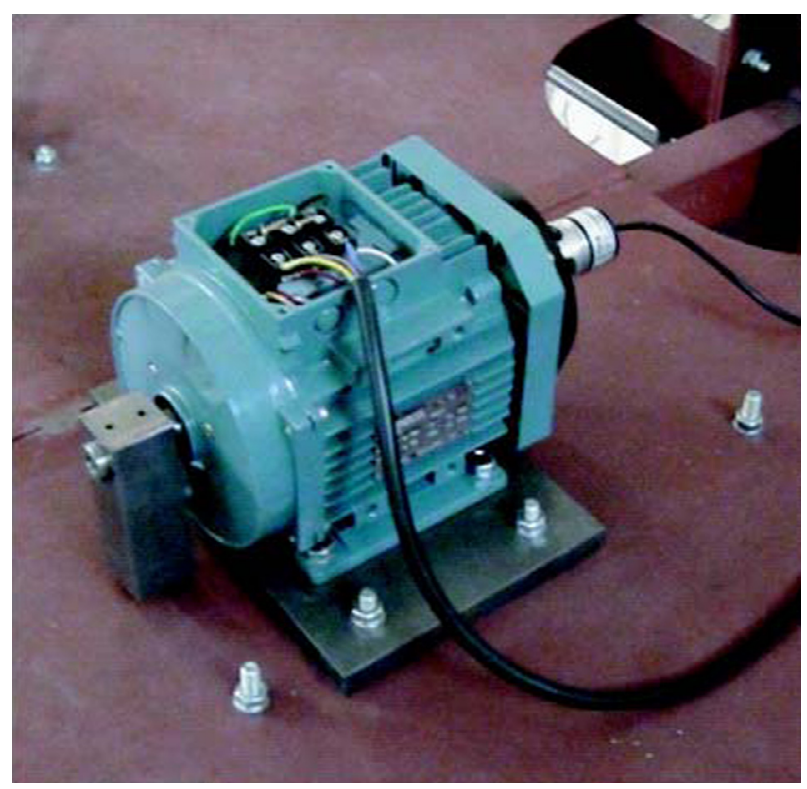

Figure 2. Pendulum exciter.

through an electronic regulator. The regulator is feedbacked by an encoder. The frequency range of the exciter is from 0 to $100 \mathrm{~Hz}$ and the angle range is from $1^{\circ}$ to $20^{\circ}$. The total mass of the pendulum is $1 \mathrm{~kg}$.
The structure was provided with four seismic Brüel \& Kæjr accelerometers with a sensibility of $10 \mathrm{~V} / \mathrm{g}$. Each was screwed at the middle of the end beam on each floor pointed to the longitudinal direction. The hardware used to record the signals was a dSPACE RTI1104 data acquisition card. The processing task was then carried out using MATLAB.

\subsection{Testing}

Free decay responses of isolated modes are chosen as experimental references for dynamic identification. This approach is very accurate because the excitation uncertainties are avoided. Moreover, free decay vibrations contain a broad range of amplitudes that shows up the nonlinearities present in the structure.

The isolation of a linear mode can be achieved by a monophase multi-point harmonic excitation in quadrature with the response at the related undamped natural frequency. This approach is known as "Appropriation" in literature [4]. The appropriation can be theoretically extended to nonlinear structures by using multi-harmonic excitation [5]. Even though this appropriation is very popular in the aerospace industry, it is not practical for in-service building applications. Instead, an imperfect appropriation consisting of a single-point mono-harmonic excitation was chosen for this case. In reference [6], it is established that this excitation isolates satisfactory a nonlinear modal mode if the structure has well-separated modes.

In the present application, the exciter was placed at the fourth floor. The experiments consisted of $20 \mathrm{~s}$ sinusoidal excitation close to the corresponding resonance followed by the free decay vibration. As accelerations are limited by serviceability conditions, only the first two bending modes could be properly excited and measured.

\subsection{Signal preprocessing}

The analogic signals were converted into digital ones at a sampling frequency of $1 \mathrm{kHz}$. Then they were filtered in order to remove a variable offset and a periodic high frequency noise present in the raw signals. Finally, signals were truncated selecting the segment corresponding to the last $65 \mathrm{~s}$ of the free decay in the first frequency and $25 \mathrm{~s}$, in the second one. The selected periods of time gave rise to a similar decrement in the response amplitude in both cases.

\section{Analytical part}

\subsection{Non-parametric identification}

The objective of this section is to choose a family of models that adequately reproduce the dynamic behaviour of the structure on the basis of the experimental results. For this purpose, the evolution of the modal properties of the structure as a function of the response amplitude is studied by means of expeditious procedures.

\subsubsection{Mode shapes}

The motion of the free vibration in the configuration space corresponding to the preprocessed time series of the acceleration 


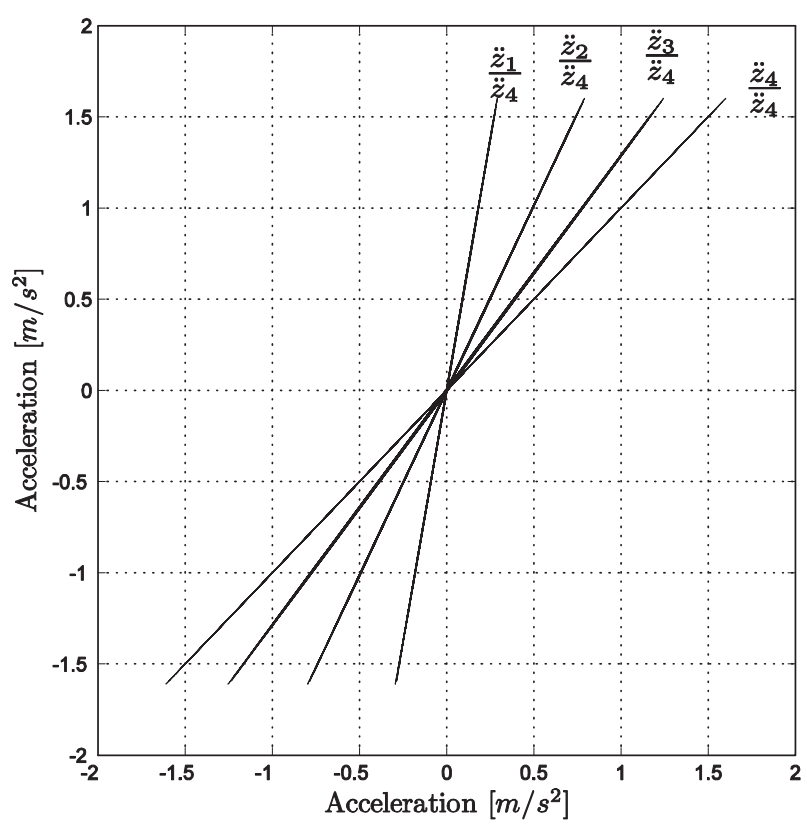

Figure 3. First mode motions in the configuration space.

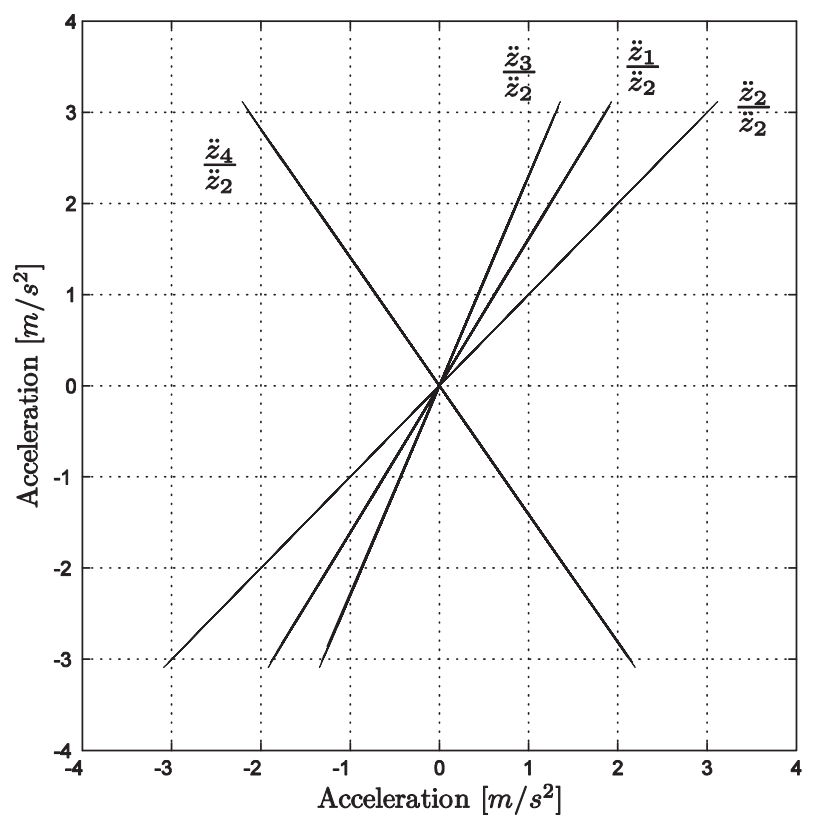

Figure 4. Second mode motions in the configuration space.

is depicted in Figures 3 and 4. As can be seen, motions exhibit a remarkable linear tend in all the cases. This means that modes do not depend on the acceleration magnitude due to the low level of energy present in the structure. Even though, as will be seen later, both stiffness and damping are weakly nonlinear, mode shapes can be considered linear within the ranges of the experiments. Taking advantage that modes are linear, the motion is defined in the model space in the rest of the paper.

\subsubsection{Stiffness and damping}

In a low-damped structure the stiffness affects mainly the period of the free vibration, while the amplitude of the response is principally governed by damping. Therefore, the study of the evolution of the period and amplitude of the free response of the structure is the key to understand its dynamic behavior. This is accomplished evaluating independently the period and amplitude of each cycle of the free response in the modal space. The evolution of the frequency as a function of the cycle amplitude is depicted in Figure 5. As can be seen, both modes show a similar trend: the higher the amplitude, the lower the frequency is. Moreover, the frequency tends to a lower limit when the amplitude increases within the range of the experiments. Under these results, an asymptotic softening law is proposed to model the structure stiffness as a function of the modal coordinate.

The damping ratio is proportional to the logarithmic decrement in low-damped structures [7]. Moreover, the logarithmic decrement is defined as the derivative of the logarithm of the amplitude of the free vibration with respect to the number of cycles. Therefore, this derivative is proportional to the damping ratio. The evolution of the amplitude of the cycles is shown in Figure 6. It is found that the logarithm of the amplitude can be expressed as a second order polynomial of the number of cycles. As a consequence of the aforementioned property, the damping ratio can be formulated as a linear function of the number of cycles. As the period of the cycles has a low variation during the free vibration, the damping ratio is approximated by a linear function of time.

\subsection{Parametric identification}

\subsubsection{Mode shapes and modal response}

The values of the mode shapes corresponding to each dof were obtained by proportional correlation of the time series in a least-squares sense. They were normalized with respect to the dof having maximum amplitude. This yields the following solution:

$$
\psi_{i}=\left(\ddot{\mathbf{x}}_{r}^{T} \ddot{\mathbf{x}}_{r}\right)^{-1} \ddot{\mathbf{x}}_{r}^{T} \ddot{\mathbf{x}}_{i}
$$

in which $\psi_{i}$ is the mode shape coordinate related to $i$-th dof, $\ddot{\mathbf{x}}_{i}$ and $\ddot{\mathbf{x}}_{r}$ represent the acceleration time series of the $i$-th and the reference dofs, respectively. Results are shown in Table 1, the correlation coefficients being close to one.

Taking advantage of the fact that modes are linear, the motion is defined in the modal space in the rest of the paper through the following least-squares solution:

$$
\ddot{\mathbf{z}}=\left(\Psi^{T} \Psi\right)^{-1} \Psi^{T} \ddot{\mathbf{X}}
$$

in which the vector $\ddot{\mathbf{z}}$ denotes the modal time series of acceleration and $\ddot{\mathbf{X}}$ is a matrix containing the spatial time series of acceleration.

\subsubsection{Stiffness and damping}

The evaluation of the model parameters of each mode is posed as the minimization of an error function accounting for the discrepancies between the numerical and experimental results. The error function is defined in the time-domain through time series of modal accelerations. The normalized mean squared error is adopted as error function 

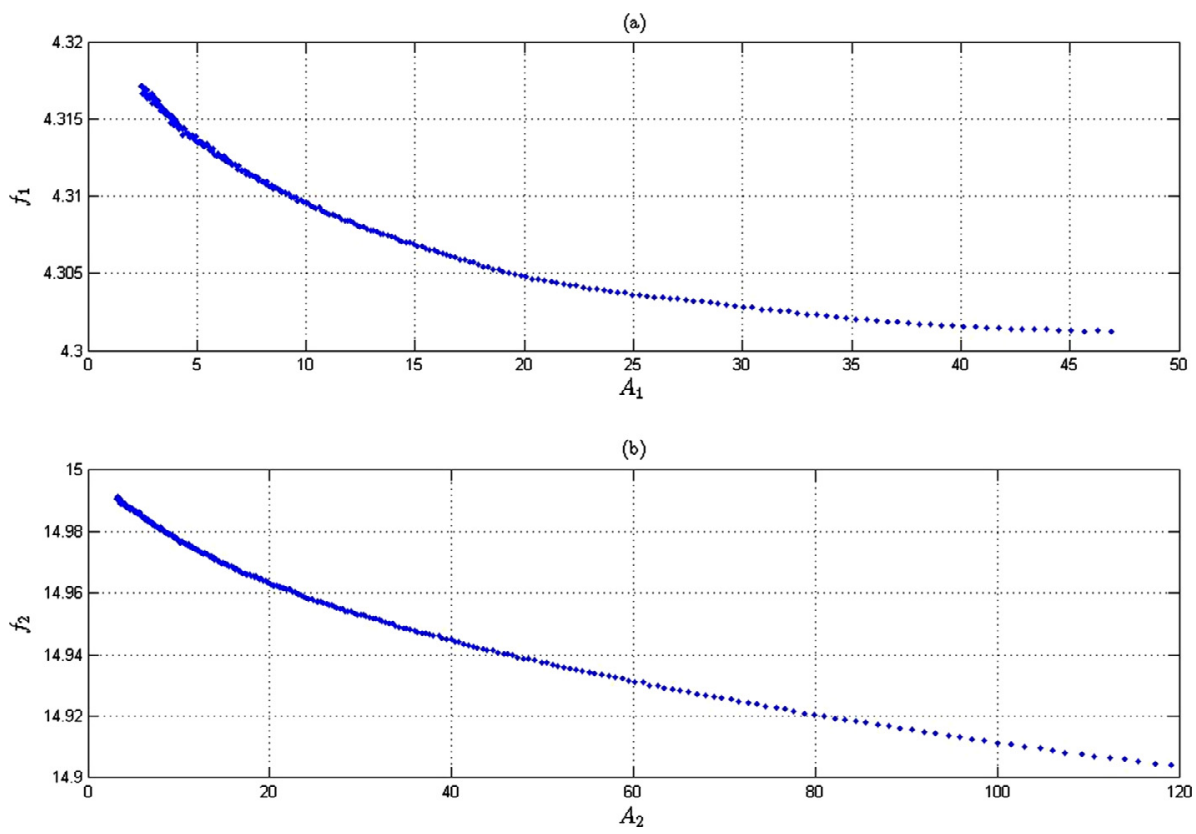

Figure 5. Frequency vs. amplitude; (a) first mode, (b) second mode.
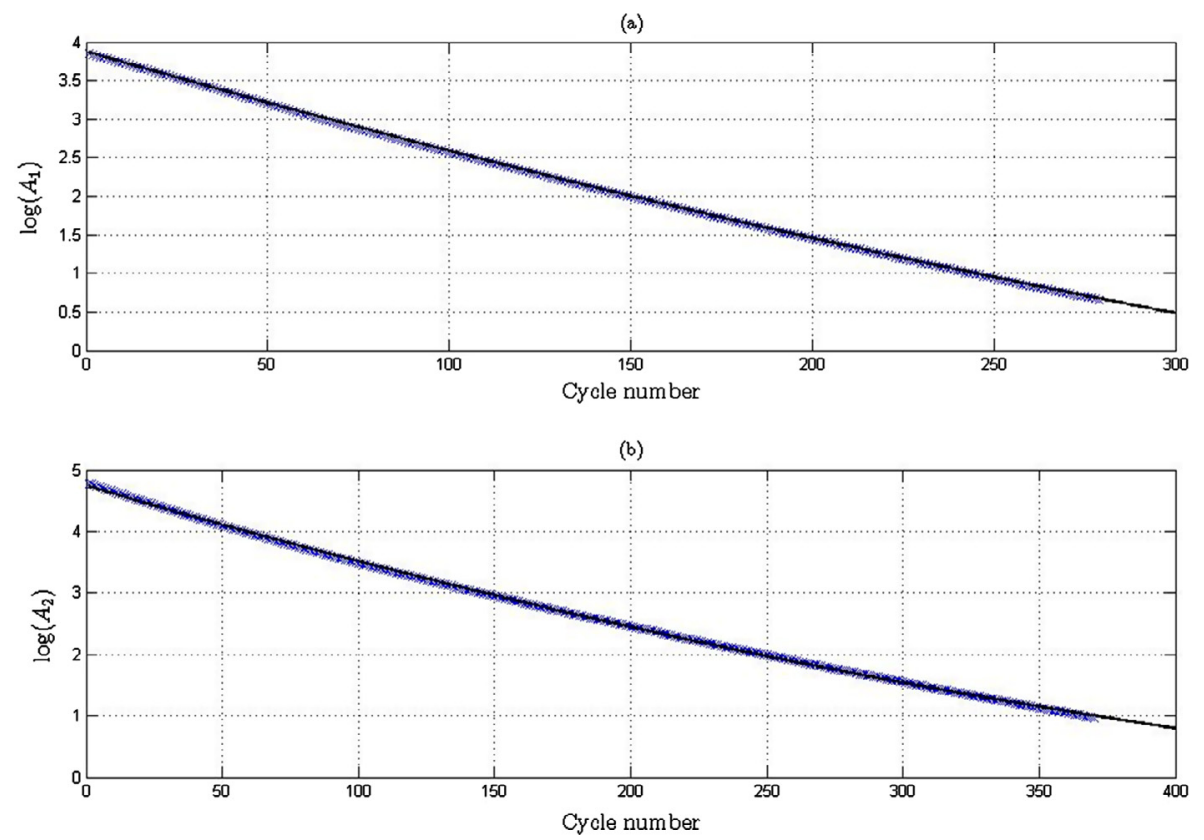

Figure 6. Log (A) vs. cycle number. $\times$ : experimental results. Line: second order polynomial fitting; (a) first mode, (b) second mode.

Table 1. Mode shapes

\begin{tabular}{lccc}
\hline Mode & dof & $\psi_{i}$ & Correlation \\
\hline 1 & 1 & 0.1811 & 0.99995725 \\
& 2 & 0.4929 & 0.99999108 \\
& 3 & 0.7767 & 0.99998806 \\
& 4 & 1.0000 & 1.00000000 \\
& 1 & 0.6186 & 0.99998222 \\
& 2 & 1.0000 & 1.00000000 \\
& 3 & 0.4326 & 0.99997735 \\
& 4 & -0.7084 & -0.99998769 \\
\hline
\end{tabular}

$$
\varepsilon=\frac{\sum_{i=1}^{M}\left(\ddot{z}_{i}^{(a)}-\ddot{z}_{i}^{(e)}\right)^{2}}{\sum_{i=1}^{M}\left(\ddot{z}_{i}^{(e)}\right)^{2}}
$$

where $\ddot{z}_{i}^{(a)}$ and $\ddot{z}_{i}^{(e)}$ represent respectively the model-predicted and the experimental modal accelerations, the latter being obtained through Equation (3). $M$ is the number of available points. The model-predicted response was numerically computed from the equation of motion assuming the following central discrete approximation for the derivatives: 
Table 2. Nonlinear softening model results.

\begin{tabular}{llllrr}
\hline Mode & $k_{1}(\mathrm{~N} / \mathrm{m})$ & $k_{2}(\mathrm{~N} / \mathrm{m})$ & $c_{1}(\mathrm{Ns} / \mathrm{m})$ & $c_{2}(\mathrm{Ns} / \mathrm{m})$ & $\alpha(\mathrm{m})$ \\
\hline 1 & 737.8597 & 728.6555 & 0.1185 & $-5.9610^{-4}$ & 0.0091 \\
2 & 8867.4 & 8680.8 & 0.4239 & $-1.0610^{-4}$ & 0.0075 \\
\hline
\end{tabular}

Table 3. Natural frequencies and fitting errors of the nonlinear model and the pure linear model.

\begin{tabular}{lcccc}
\hline Model & $f_{r 1}(\mathrm{~Hz})$ & $\varepsilon(\%)$ & $f_{r 2}(\mathrm{~Hz})$ & $\varepsilon(\%)$ \\
\hline Linear & 4.3048 & 1.8082 & 14.9316 & 4.9881 \\
Nonlinear & 4.3232 & 0.0047 & 14.9871 & 0.0136 \\
\hline
\end{tabular}

$$
\begin{gathered}
\dot{z}(t)=\frac{z(t+\Delta t)-z(t-\Delta t)}{2 \Delta t} \\
\ddot{z}(t)=\frac{z(t+\Delta t)-2 z(t)+z(t-\Delta t)}{\Delta^{2} t}
\end{gathered}
$$

where $\Delta t$ represents the integration period. A value of $\Delta t=5 \times 10^{-4} \mathrm{~s}$ was chosen for the integration. This value constitutes a trade-off between accuracy of results and computation cost.

The minimization is carried out by an adaptive stochastic algorithm developed previously by the authors, which will be referred to as Beta algorithm for the remainder of the paper. This algorithm is described in detail and tested against numerical simulations and experiments in reference [8]. A brief description of the Beta algorithm is given here. In this algorithm the solution is sought by sampling the parameters to be identified within a bounded space in an iterative form. The Beta distribution, which is consistent with the bounded character of the search space, is selected for sampling purposes. In each step, the parameters of the distributions are modified accordingly the results of the previous steps. The process is repeated until a given stopping criterion is reached.

Accordingly to the results of the non-parametric identification, the following time-variant linear law is proposed to model the modal damping coefficient

$$
c_{r}=c_{r 1}+c_{r 2} t
$$

in which $c_{r 1}$ and $c_{r 2}$ are parameters to be identified and $t$ stands for time.

The following nonlinear function adapted to the empirical results is proposed to model the modal stiffness:

$$
k_{r}(t)=\frac{k_{r 1} \alpha+k_{r 2}\left|z_{r}(t)\right|}{\alpha+\left|z_{r}(t)\right|}
$$

in which $k_{r 1}$ and $k_{r 2}$ are the bounds of the stiffness and $\alpha$ is a shape parameter. The function is symmetric with respect to the modal coordinate $z_{r}$. Moreover, its value is $k_{r 1}$ for $z_{r}=0$ and is asymptotic to $k_{r 2}$ when $z_{r}$ tends to infinity; therefore, $k_{r 1}$ represents the stiffness of the underlying linear model. The decrement of the function diminishes when the parameter $\alpha$ increases.

The equation of motion becomes

$$
m_{r} \ddot{z}_{r}(t)+\left(c_{r 1}+c_{r 2} t\right) \dot{z}_{r}(t)+\left(\frac{k_{r 1} \alpha+k_{r 2}\left|z_{r}(t)\right|}{\alpha+\left|z_{r}(t)\right|}\right) z_{r}(t)=0 \text {. }
$$

The central differences scheme yields the following solution of the modal response:

$$
\begin{aligned}
z_{r}(t)= & \frac{\left(\frac{2}{\Delta^{2} t}-\frac{k_{r 1} \alpha+k_{r 2} \mid z_{r}(t-\Delta t)}{\alpha+\left|z_{r}(t-\Delta t)\right|}\right) z_{r}(t-\Delta t)}{\frac{1}{\Delta^{2} t}+\frac{c_{r 1}+(t-\Delta t) c_{r 1}}{2 \Delta t}} \\
& -\frac{\left(\frac{1}{\Delta^{2} t}-\frac{c_{r 1}+(t-\Delta t) c_{r 2}}{2 \Delta t}\right) z_{r}(t-2 \Delta t)}{\frac{1}{\Delta^{2} t}+\frac{c_{r 1}+(t-\Delta t) c_{r 2}}{2 \Delta t}} .
\end{aligned}
$$

The total number of unknown parameters is five in this case. They were achieved through the Beta algorithm. Results are shown in Table 2. The corresponding frequencies of the underlying linear model and those of a pure linear model are shown in Table 3 along side the fitting errors. As can be seen, the fitting errors of the nonlinear model are almost three orders of magnitude lower than those of the pure linear model. This indicates that the nonlinear model is much more precise describing the response of the model. As for the natural frequencies, the discrepancies are around $0.4 \%$ in both modes.

\section{Conclusions}

This study of the Uniovi structure allows a number of conclusions to be drawn. In the experimental part, it was found that the first two bending modes can be individually isolated by means of a single-point mono-harmonic excitation placed at the top floor and remain during the subsequent free decay vibration. The analysis of the motions of the free decays reveals that mode shapes are linear within the acceleration ranges of the experiments. An exploratory study of the evolution of the period and amplitude of the free decay cycles was essential to infer the nonlinearities present in the structure. It was found that the modal damping varies lineally in time and the modal stiffness varies asymptotically as a function of the modal amplitude. The adopted nonlinear model was calibrated by minimizing the discrepancies between the model-predicted responses and the experiments in the time domain. The fitting error of the proposed nonlinear model is near three orders of magnitude lower than that of a pure linear model. A discrepancy around $0.4 \%$ was found between the natural frequencies of the pure linear model and those of the linear model underlying the nonlinear one.

Acknowledgements. The authors wish to thank the Science and Innovation Ministry of Spain for its financial support (Projects BIA2006-15266-C02-01 and BIA2006-15266-C02-02). The experimental part of this work has been performed at the laboratory of Fluid Mechanics of the University of Oviedo. The collaboration of the staff of this area, particularly Professor Santolaria, is greatly acknowledged. 


\section{References}

1. Rosenberg RM. 1966. On nonlinear vibrations of systems with many degrees of freedom. Advances in Applied Mechanics, 9, $155-242$.

2. Kerschen G, Peeters M, Golinval J-C, Vakakis AF. 2009. Nonlinear normal modes, Part I: A useful framework for the structural dynamicist. Mechanical Systems and Signal Processing, 23, 170-194.

3. Peeters M, Viguié R, Sérandour G, Kerschen G, Golinval J-C. 2009. Nonlinear normal modes, Part II: Toward a practical computation using numerical continuation techniques. Mechanical Systems and Signal Processing, 23, 195-216.

4. Ewins DJ. 2000. Modal testing: theory, practice and application. Baldock, Hertfordshire: Research Studies Press Limited, ISBN: 978-0863802188.
5. Peeters M, Kerschen G, Golinval J-C. 2011. Modal testing of nonlinear vibrating structures based on nonlinear normal modes: Experimental demonstration. Mechanical Systems and Signal Processing, 25, 1227-1247.

6. Peeters M, Kerschen G, Golinval J-C. 2011. Dynamic testing of nonlinear vibrating structures using nonlinear normal modes. Journal of Sound and Vibration, 330, 486-509.

7. Maia JM, Silva JMM. 1997. Theoretical and experimental modal analysis. Baldock, Hertfordshire: Research Studies Press Limited., ISBN: 978-0863802089.

8. Zapico-Valle JL, Alonso-Camblor R, González-Martínez MP, García-Diéguez M. 2010. A new method for finite element model updating in structural dynamics. Mechanical Systems and Signal Processing, 24, 2137-2159.

Cite this article as: Zapico-Valle JL \& García-Diéguez M: Dynamic modeling and identification of the Uniovi structure. Int. J. Simul. Multisci. Des. Optim., 2014, 5, A06. 